\title{
SUPPORTING VIDEO IN HETEROGENEOUS MOBILE ENVIRONMENTS
}

\author{
Nicholas Yeadon, Nigel Davies, Adrian Friday, Gordon Blair \\ Lancaster University, Lancaster, UK, LA1 4YR \\ \{njy, nigel, adrian, gordon\}@comp.lancs.ac.uk
}

KEYWORDS: H263, VLBR Video, Heterogeneity, Overlay Networks.

\begin{abstract}
High quality digital wireless networks and advances in multimedia compression schemes now permit the transmission of video streams over mobile networks. This opens up video communications to a new range of mobile users. We present our experiences of integrating very-low-bit-rate video encoding into a heterogeneous mobile environment as part of an ongoing project to provide multimedia support for the emergency services. This paper focuses on the features required to enable open working between a variety of applications, end-systems and networks and the performance of a very low bit rate encoder.
\end{abstract}

\section{INTRODUCTION}

Advances in digital wireless technologies and improved multimedia compression algorithms are enabling a convergence of high requirement applications and low capability networking. The higher throughput and lower error rates of the new range of digital wireless networks such as GSM [11], WaveLAN [13], and the emerging TETRA [4] private mobile radio standard provide the opportunity to deliver low frame rate compressed video to mobile users.

In parallel, improvements in processor power have enabled many mobile computer systems to be able to source and sink multimedia streams. This can be attributed to these powerful computers being able to perform complex and processor intensive compression functions, the emergence of specialised codec hardware and advances in the development and standardisation of compression algorithms. Currently under standardisation are, the recently completed, H.263 [9] and, in final working draft, MPEG 4 [7] very low bit rate (VLBR) video compression schemes. These compression algorithms are intended for bit-rates less than 64Kbit/s.

This paper describes a prototype system developed within our laboratory which provides video conferencing and remote surveillance functionality to mobile users equipped with pen based tablets and GSM, TETRA or WaveLAN based networking. This prototype has been designed as part of a project to develop a new range of services and collaborative applications for the future users of the recently standardised TErrestrial (formerly Trans European) Trunked RAdio system (TETRA), namely the emergency services, customs officials, utility workers and delivery and freight organisations. The system we have developed demonstrates the initial possibilities of the new technology.

Together with a detailed description of the application prototype the paper also describes the supporting infrastructure which addresses the technical issues of interworking between fixed and wireless users, video transmission and late entry, i.e. entering an already established data flow. Finally, the paper provides performance measurements and reports on our practical experiences of using H.263 in a heterogeneous mobile environment.

\section{MOTIVATION}

We are currently undertaking a project to develop mobile multimedia services and applications for members of the emergency services [10]. As part of this work we have conducted a requirements study which has identified the following key requirements:

- Establishment and management of multiple dynamic groups.

- Multiparty integrated voice and data transmission.

- Exchange of multimedia information, including still images, slow-scan and potentially real-time video.

- Interoperability with other agencies and Emergency Services.

- Support for interworking in heterogeneous architectures.

- Security of data and physical end-systems.

- High levels of availability and dependability.

- $\quad$ Automated locality and status reporting.

These requirements can be illustrated by considering the following application scenario in which a major incident such as a bank robbery is taking place. In an ideal world, where all systems are open and interoperable, the police would be able to patch directly into the surveillance cameras within the bank from their control centre using high-speed land-lines. The video images from these cameras could then be transmitted via appropriate interworking units (between the fixed and wireless network) to police en route and attending the incident. The officer(s) co-ordinating the operation could also control the camera from which these images originate (e.g. panning or tilting the camera). The control centre would be able to capture an image of one of the robbers faces that they might relay in digital form directly to police headquarters for identification.

The technology to realise such a scenario is now available, but a significant remaining challenge is to integrate these 
technologies in a heterogeneous environment. In the above example, high quality MPEG 1 or 2 compressed video may be transmitted via a high speed ATM connection between the bank and the Police control centre. The control centre may utilise high capability workstations and mainframes as endsystems to analyse and process the video stream. From there low quality H.263 or MPEG 4 video may be transmitted via GSM or TETRA to mobile PCs at the scene of the crime. Once inside the bank, special response units may be able to receive better quality video on head up displays transmitted to them via the building's own wireless LAN (e.g. WaveLAN). This scenario could quite easily be transposed to a number of different domains each having the same characteristics of a group of users with disparate end-systems dynamically roaming across different capability overlay networks accessing a multitude of information objects, (e.g. a tourist application [5]).

We have designed a demonstration system based on the scenario above that exploits current technology to deliver compressed video at speeds and quality suitable for a range of wired and wireless networks. The next section gives an introduction to the encoding scheme used to compress the video in the prototype system, namely H.263.

\section{H.263 VIDEO COMPRESSION STANDARD}

In the application prototype described in the next section we have made use of the freely available H.263 encoding and decoding algorithms from Telenor R\&D [1]. The following section describes the key features of $\mathrm{H} .263$ relevant to the work presented here. Some knowledge of existing compression schemes is assumed.

The H.263 standard is not radically different from previous video compression standards such as MPEG 1 [6] and H.261 [8]; the main element of H.263 is still a block based discrete cosine transform (DCT). In many ways H.263 can be viewed as an enhanced or optimised H.261 scheme. The main differences lie in more efficient ways of coding DCT coefficients (with improved adaptive Variable Length Code (VLC) tables), improved entropy encoding and increased precision motion compensation.

\subsection{Overview of $\mathbf{H . 2 6 3}$}

In common with H.261, H.263 has two main frame types. In additonal to H.261 it also has an optional third frame type. The two primary frames are either intra-coded frames (I-frames), encoded entirely without reference to any other frames, and inter-coded or predictive frames (P-frames), predicted off intracoded reference frames. The third type of frame is derived from MPEG bidirectionally predicted frames (B-frames) called a PB frame. A PB-frame is two pictures coded as one unit. The frequency at which I-frames are placed into a stream affects the resilience to the more catastrophic errors, such as total frame losses; in effect a new I-frame flushes the decoding process.

\begin{tabular}{|l:l:l:l:l|}
\hline $\begin{array}{l}\text { Picture } \\
\text { Format }\end{array}$ & lum & lum & chrom & lhrom \\
peline of lines & pels/line & $\begin{array}{l}\text { no } \\
\text { lines }\end{array}$ \\
\hline sub-QCIF & 128 & 96 & 64 & 48 \\
\hdashline QCIF & 176 & 144 & 88 & 72 \\
\hdashline CIF & 352 & 288 & 176 & 144 \\
\hline
\end{tabular}

\begin{tabular}{|c|c|c|c|c|}
\hline $4 \mathrm{CIF}$ & 704 & 576 & 352 & 288 \\
\hline $16 \mathrm{CIF}$ & 1408 & 1152 & 704 & 576 \\
\hline
\end{tabular}

Table 1: H.263 standardised image formats

As with H.261, H.263 has a fixed set of frame sizes that can be encoded. H.263 has five image size formats: sub-QCIF (sub Quarter Common Interchange Format), QCIF, CIF (optional), 4CIF (optional), and 16CIF (optional). In comparison H.261 only supports Q-CIF and the optional CIF size images. The various image sizes (for the luminance and chrominance components) are shown in Table 1 [9].

\subsection{Symmetry of Coding}

The H.263 codec has been designed to be relatively symmetrical, in that the encoding process should not be significantly more complex or processor intensive than the decoding process. This is to ensure its suitability for bidirectional visual communication (i.e. video conferencing).

\subsection{Resilience}

An important issue when considering H.263 for wireless communications is its sensitivity to bit-errors. Obviously as a video stream is compressed each bit becomes more important and the consequence of a single bit-error is of greater significance. There is a trade-off between the amount of redundancy that can be removed from a stream to compress it and the amount of redundancy that has to be added to the stream to protect it from bit-errors. An interworking gateway (see Section 4.3) could conceivably add and remove FEC information as video streams enter and leave a noisy environment, respectively.

\section{INTEGRATED TESTBED}

This section describes our experimental architecture and its component parts. This includes the infrastructure, a capture, compression and delivery system, an interworking gateway and a video transmission protocol.

A prototype surveillance application has been developed to demonstrate the various components of the system. The application allows groups of users connected by both fixed and wireless networks to receive video from multiple surveillance cameras. The surveillance cameras are connected to the infrastructure using a range of networking technologies (see Section 4.1).

\subsection{Infrastructure}

Our experimental infrastructure is shown in Figure 1. The system consists of a number of low-cost cameras (Connectix QuickCams, denoted by (2) mounted in a surveillance capacity connected to fixed and wireless end-systems. These end-systems perform video compression functions and act as video stream sources. 


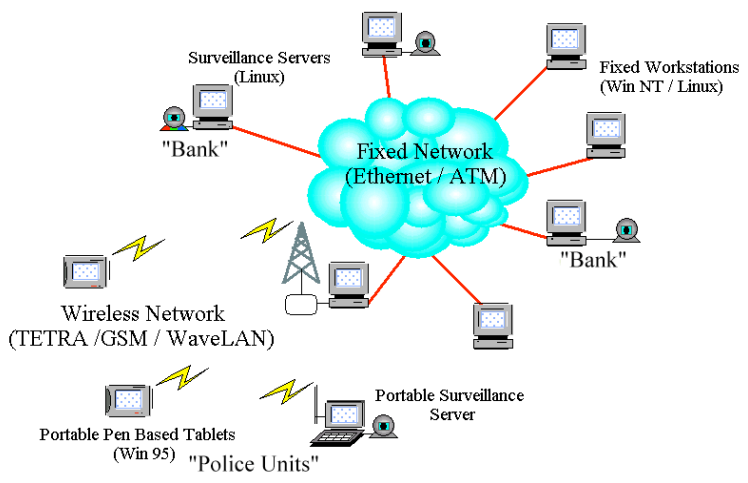

Figure 1: Experimental Infrastructure

The various machines are connected via a range of ethernet and ATM fixed networks and WaveLAN and GSM networks. We also are able to emulate a TETRA network using a public domain wireless network emulator [3] while awaiting prototype TETRA equipment.

\subsection{Video Capture and Control}

The surveillance application employs the Telenor R\&D H.263 codec software [1]. The original codec software produced H.263 files from a raw video file. The H.263 encoding software has been substantially altered to include source capture directly from a camera, dynamic remote control of the encoding parameters, packetisation of individual compressed frames and rate paced transmission. A number of optimisations have been made to improve compression time. For example, if the encoder knows the camera only produces $\mathrm{B} \& \mathrm{~W}$ images then the encoding of the chrominance information can be performed more rapidly. The decoder has also been significantly altered to issue connection requests, read compressed video off the network (instead of from a file) monitor its reception quality, and issue feedback control messages to source and interworking units. The encoding software currently runs on Linux based PCs while the decoder operates in both Linux and Windows NT environments.

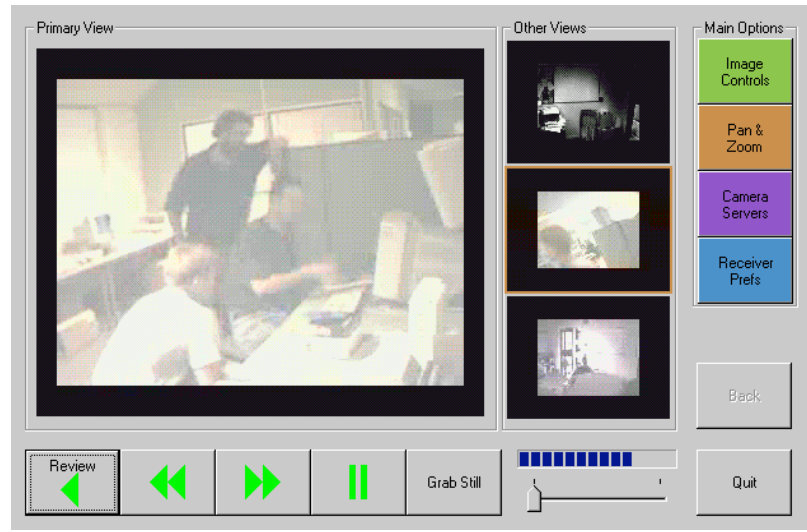

Figure 3: Screen shot of surveillance application

The video source encoder can be controlled by any receiver that has the correct permissions. Feedback messages are used to control typical camera adjustments, such as brightness, contrast, etc., and also to zoom and pan the image electronically. The zoom function allows a much larger image to be captured at source and the recipient to receive only the area of the picture of interest. A much smaller image is therefore transmitted so reducing the bandwidth requirement.
The surveillance application allows a number of streams to be received and displayed from separate encoders. Each stream is transmitted and displayed as a sub-QCIF until the user selects a stream which may be of interest. The video stream is then displayed in the main, larger, window. The transmitted stream will then be adjusted in accordance with the user's preferences. For example, preference options include whether to switch to a faster frame rate or to encode the frames with a higher resolution and screen size.

\subsection{Interworking}

Conflicts in requirements will occur when there is a group of heterogeneous receivers within the same multipeer communication session. For example, a mobile receiver within a multicast group may be experiencing high packet loss and so signal the source to reduce its transmission rate, whereas within the same session another receiver fixed to a wired network may be receiving a perfect transmission and wish to increase the frame rate. This has been reported as the multicasting problem [14]. For these reasons we have a distinct interworking unit, which acts as a gateway between wired and fixed networks, performing filtering [14] and adaptive functions where necessary.

Our previous work has developed a number of filtering mechanisms that can dynamically adapt MPEG 1 and JPEG compressed video streams [14]. This dynamic adaptation is performed not at the source but at key locations within a multipeer dissemination tree called filter servers. Filter agents on each filter server perform operations on the compressed or semi-uncompressed streams (i.e. operations are applied to the run-length coded DCT coefficients to achieve fast and reactive processing). Typical filtering mechanisms include frame dropping, low-pass filtering, colour-reduction, requantization and transcoding.

Some of these filtering mechanisms have been integrated into the present surveillance system and perform operations on H.263 compressed video. This permits users on a fixed highspeed network to receive full frame-rate high quality video while other users on low-speed wireless networks may still participate in the same communication session but receive lower rate and quality images. Currently under development is an MPEG 1 to H.263 transcoder to enhance interworking functionality.

The interworking gateway is used to filter selected types of packets. If a high number of packets are being lost by the mobile system then this will produce a corrupt and distorted video sequence (as many frames are reliant on other frames being decoded). In such a case the surveillance application can instruct the filtering gateway to discard all frames but the Iframes. This will reduce the bit-rate of the stream and produce a stream that is more resilient to errors, since each frame is independently encoded. Of course the frame is reduced but the image quality will remain high. This does not affect the other recipients on the fixed network.

\subsection{Video Transmission Scheme}

Video transmission is achieved by using the Continuous Media Protocol (CMP) [14] over the fixed network and a MobileCMP over the wireless network. It includes an Application Level Framing (ALF) [2] media assembly format (above UDP) and a rate control mechanism. As all communication is via datagrams, connection state is maintained at the application layer, i.e. at source agents, filter agents and client applications. 
Like the IETF Real-time Transport Protocol (RTP) [12] the CMP protocol is embedded into the application.

The packetisation strategy for the Continuous Media Protocol is illustrated in Figure 4. This also shows the standard UDP header. In the CMP header there are three important fields: the payload type, the sequence number and the time stamp.

The payload type specifies the media type and also sub-types of that media. For example, the H.263 video media type used in the prototype system consists of three sub-types: I-picture, Ppicture and PB-picture. The sequence number identifies the transmission order of the packets in the data stream. It can be used to detect lost packets or out-of-order packets. The time stamp is the local time (in $\mu$ secs) at the source when the packet was generated. Again, it can be used for detecting lost or delayed packets and also for resynchronising incoming packets at the end-system.

In the Mobile-CMP packetisation scheme the packet header is reduced to 1 or 2 bytes and a selective re-transmission scheme is used to enable the protocol to adapt to different media types' QoS requirements.

\begin{tabular}{|c|c|c}
\hline 16-bit Src Port No. & 16-bit Dest Port No. \\
\hline 16-bit UDP Length & 16-bit UDP Checksum & UDP \\
\hline 16-bit Payload Type & 16-bit Pad \\
\hline 32-bit Sequence No. & \\
\hline 32-bit Time Stamp \\
Header
\end{tabular}

Figure 4: Packetisation of Continuous Media Data

There are two choices of rate control. The first and simplest is a frame oriented rate control. That is, the frames are transmitted from the source at regular intervals specified by the required frame rate. This method maintains the isochrony of the media stream, but a potentially bursty traffic shape is produced.

The second choice of rate control is implemented around a credit-based flow control scheme. This method attempts to achieve a constant data rate from the source. An output stream is allocated a credit token, initially set to a level corresponding to the data rate required. When a packet is transmitted, the credit is reduced by an amount related to the size of packet. The credit then increases over time at a rate associated with the data rate required. A packet can only be transmitted if there is enough credit available for that size of packet. This allows small packets to be transmitted frequently and larger packets to be delayed until there are resources available. This method produces a consistent flow of data but has the disadvantage of introducing inter-frame jitter before the data has even left the source.

The actual rate control and rate to use is specified by the sending entity. This rate can be dependent on information gleaned from the receivers.

The tagging of each packet with a sequence number and timestamp allows each receiver to monitor the QoS it is receiving, i.e. it can detect packet loss, monitor data rates, packet jitter, etc. By using this information the receivers can issue adaptation signals back to the source which may include rate control or encoding parameter adjustments (e.g. changing the source encoding parameters).

\subsection{Late entry}

In a group communication it is typical for calls to be set up by a small number of users and for other recipients to join the group session at a later time. This is called 'late entry'. When transmitting compressed video where individual frames are predicted off other frames there is an inter-dependency between packets. This means that a user entering a group late may need reference frames that have already been transmitted. Without these reference frames the connection must wait until the next independent object is transmitted, e.g. an intra coded reference frame. In some compression schemes, such as MPEG, other information is needed to initialise the decoder. This information is carried in Sequence Headers and transmitted very infrequently, sometimes only once. Fortunately, the H.263 I-frame picture header contains all the information necessary to initialise a decoder.

If late entry is a desired attribute of an H.263 based communication session then there are three approaches: Iframes must be transmitted relatively frequently and the user must wait for then next reference to be transmitted, the last reference frame and any frames since may be transmitted again to a joining client to allow it to 'catch up', or the encoder may be forced to generate a new I-frame. The first option may result in a larger stream over time due to the increased number of Iframes. The second and third options may cause a large burst when a client joins a group but it has the advantage that larger I-frames can be transmitted infrequently. The second option implies there must be an object which caches previous frames and the decoder must be able to decode faster than the current stream is transmitting (or else it would never catch up).

Late entry is especially desirable in mobile environments because it allows quick recovery from burst errors, losses and connection drop outs that may occur during handovers between cells and different overlay networks.

The choice of schemes is dependent upon how dynamic a group is (i.e. how often clients join and leave a group) and how long users are prepared to wait to start receiving video once connected. The emergency services have stringent requirements relating to the maximum mobile call set-up time in emergency situations (less than a quarter of a second for voice traffic). As video communications becomes more common-place in this environment this may impinge on the schemes used for video transmission and encoding.

\section{PERFORMANCE EVALUATION AND ANALYSIS}

We have conducted a performance evaluation of our system focusing on the H.263 encoder and decoder. The evaluation procedure used a $100 \mathrm{MHz}$ Pentium running the LINUX operating system to capture images from a greyscale Connectix Quickcam camera and then compress and transmit the video stream over WaveLAN to a mobile pen based mobile 486 DX2 PC running Windows 95. The tests were performed with the encoder in a variety of settings. The image sizes used were Sub-QCIF, QCIF and CIF (see Section 3.1). The quantization factor (which basically sets the image-quality-to-compression ratio) was set to very high image quality $(\mathrm{q}=1)$, average/normal use $(q=10)$ and low quality $(q=31)$. The I-frame frequency was 
set to 1 in 10 and 1 in 100 frames. The encoder was also tested with different input streams from the camera; the camera was left stationary with no movement in the scene at all (in a surveillance application possibly monitoring a warehouse this would be the normal mode of operation) and the camera was made to pan by physically oscillating from side to side at a rate of $7.5^{\circ} / \mathrm{sec}$ (not to be confused with the software pan and zoom described earlier).

An important reasoning behind the evaluation stages was to test the system in a real operational environment with varying light conditions, random events, etc. The results predict a best case scenario where the camera is continually monitoring the same unchanging (though relatively complex) scene and worst case, where a camera is panning with potentially (depending on the pan period) a different image in every frame. The figures quoted are averages of measurements taken at different times over a two week trial period.

Table 2 shows the speed at which the H.263 encoder can capture, compress and transmit frames (running on a $100 \mathrm{MHz}$ Pentium P100) and the associated bit-rate this produces. This is when the camera is stationary.

\begin{tabular}{|l|l:l|l:l}
\hline Q=10 & I-frame $=1 / 10$ & I-frame $=1 / 100$ \\
Image Size & $\begin{array}{l}\text { Frame } \\
\text { Rate }\end{array}$ & $\begin{array}{l}\text { Bit } \\
\text { Rate }\end{array}$ & $\begin{array}{l}\text { Frame } \\
\text { Rate }\end{array}$ & $\begin{array}{l}\text { Bit } \\
\text { Rate }\end{array}$ \\
\hline Sub-QCIF & $2.4 \mathrm{fps}$ & $3.2 \mathrm{Kbit} / \mathrm{s}$ & $2.2 \mathrm{fps}$ & $947 \mathrm{bit} / \mathrm{s}$ \\
\hdashline QCIF & $1.2 \mathrm{fps}$ & $4 \mathrm{Kbit} / \mathrm{s}$ & $1.2 \mathrm{fps}$ & $631 \mathrm{bit} / \mathrm{s}$ \\
\hdashline $\mathrm{CIF}$ & $0.29 \mathrm{fps}$ & $2.1 \mathrm{Kbit} / \mathrm{s}$ & $0.33 \mathrm{fps}$ & $422 \mathrm{bit} / \mathrm{s}$ \\
\hline
\end{tabular}

Table 2: Frame Rate to Bit Rate (stationary camera)

Table 2 shows that the encoding process is relatively heavyweight. In fact, in high-speed networks the limiting factor is the time it takes to encode each frame and not the network throughput. Obviously this is less of an issue in low-speed wireless networks. It can be seen from Table 2 that the frequency at which an I-frame is placed into the data stream severely affects the bit-rate. I-frames are much larger than predicted frames (P-frames) but provide increased error resilience and also reduce the time a user may have to wait for late entry (see Section 4.5). This is of greater significance when the image has little movement since much of the information can be predicted from previous frames. The I-frames also prevent a gradual build up of decoding quality losses in such a case.

The slightly higher frame rate in Tables 2 and 3 when the Iframes are more frequent are caused by the reduced amount of processing needed to encode I-frames and hence, as the network is not the bottleneck, this results in a higher encoding rate.

\begin{tabular}{|c|c|c|c|c|c|c|c|}
\hline \multirow{2}{*}{$\begin{array}{l}\mathrm{Q}=10 \\
\text { Image }\end{array}$} & \multicolumn{2}{|c|}{$\mathrm{I}$-frame $=1 / 10$} & \multicolumn{2}{|c|}{$\mathrm{I}$-frame $=1 / 100$} & & \multicolumn{2}{|c|}{$\begin{array}{l}\text { Table 5: Frame Rate to Bit Rate (panning camera) } \\
\text { Q-Scales }\end{array}$} \\
\hline & Frame & Bit & Frame & Bit & & & \\
\hline & \multirow{2}{*}{\multicolumn{2}{|c|}{ Profiles }} & \multirow{2}{*}{\multicolumn{2}{|c|}{ Max Data Rate }} & \multicolumn{3}{|c|}{ Expected frame rate (panning camera) } \\
\hline & & & & & Sub-QCIF & QCIF & CIF \\
\hline
\end{tabular}

\begin{tabular}{|l|l:l|l:l} 
Size & Rate & Rate & Rate & Rate \\
\hline $\begin{array}{l}\text { Sub- } \\
\text { QCIF }\end{array}$ & $1.2 \mathrm{fps}$ & $3.3 \mathrm{Kbit} / \mathrm{s}$ & $1.1 \mathrm{fps}$ & $2.8 \mathrm{Kbit} / \mathrm{s}$ \\
\hdashline QCIF & $0.48 \mathrm{fps}$ & $3.3 \mathrm{Kbit} / \mathrm{s}$ & $0.5 \mathrm{fps}$ & $2.7 \mathrm{Kbit} / \mathrm{s}$ \\
\hdashline $\mathrm{CIF}$ & $0.12 \mathrm{fps}$ & $2.5 \mathrm{Kbit} / \mathrm{s}$ & $0.12 \mathrm{fps}$ & $1.65 \mathrm{Kbit} / \mathrm{s}$ \\
\hline
\end{tabular}

Table 3: Frame Rate to Bit Rate (panning camera)

Table 3 show the case when the camera is panning. Obviously the bit-rate produced is higher as there is significant movement in the scene. Also, the additional processing required to encode the changes results in a slower frame rate. The performance when encoding the larger and slower pictures is made worse because the camera is oscillating quite fast in relation to the frame rate, hence the encoder will see this a much faster motion. In fact, the image is usually completely different from the previous image.

The optional extensions described in the annexes of the H.263 standard, were also tested. Surprisingly, the additional features did not slow down the encoding process significantly. However, the bit-rate was reduced for each of the three different size images as shown in Table 4.

\begin{tabular}{|l|l|l|}
\hline Q=10 & I-frame $=1 / 10$ & \\
\cline { 2 - 3 } Image Size & Frame Rate & Bit-Rate \\
\hline Sub-QCIF & $1.1 \mathrm{fps}$ & $2.2 \mathrm{Kbit} / \mathrm{s}$ \\
\hdashline QCIF & $0.5 \mathrm{fps}$ & $2.2 \mathrm{Kbit} / \mathrm{s}$ \\
\hdashline $\mathrm{CIF}$ & $0.12 \mathrm{fps}$ & $1.9 \mathrm{Kbit} / \mathrm{s}$ \\
\hline
\end{tabular}

Table 4: Frame Rate to Bit Rate (panning camera with options Annex D, E \& F)

The final set of results shows the effect of using different quantization scales (quality factor). The previous results used the quantization parameter set to the default value 10 (average quality). Table 5 shows the large increase in bit-rate if the highest quality encoding is used. The frame rate also increases slightly when encoding with the highest quality as the processor does not have to quantize each DCT value, i.e. there is less processing perform.

\begin{tabular}{|l|l:c|}
\hline Image size=QCIF & I-frame $=1 / 10$ & \\
\hline Quantization Scale & Frame Rate & Bit-Rate \\
\hline $\mathrm{Q}=1$ & $0.59 \mathrm{fps}$ & $56 \mathrm{Kbit} / \mathrm{s}$ \\
\hdashline $\mathrm{Q}=10$ & $0.48 \mathrm{fps}$ & $3.3 \mathrm{Kbit} / \mathrm{s}$ \\
\hdashline $\mathrm{Q}=31$ & $0.46 \mathrm{fps}$ & $2.7 \mathrm{Kbit} / \mathrm{s}$ \\
\hline
\end{tabular}

Table 5: Frame Rate to Bit Rate (panning camera) different Q-Scales 


\begin{tabular}{|l|l|l|l|l|}
\cline { 3 - 5 } GSM & $9.6 \mathrm{Kbit} / \mathrm{s}$ & $3.7 \mathrm{Fps}$ & $1.8 \mathrm{Fps}$ & $0.68 \mathrm{Fps}$ \\
\hline TETRA 1 slot & $7.2 \mathrm{Kbit} / \mathrm{s}$ & $2.8 \mathrm{Fps}$ & $1.3 \mathrm{Fps}$ & $0.5 \mathrm{Fps}$ \\
\hline TETRA 2 slot & $14.4 \mathrm{Kbit} / \mathrm{s}$ & $5.7 \mathrm{Fps}$ & $2.7 \mathrm{Fps}$ & $1 \mathrm{Fps}$ \\
\hline TETRA 3 slot & $21.6 \mathrm{Kbit} / \mathrm{s}$ & $8.5 \mathrm{Fps}$ & $4 \mathrm{Fps}$ & $1.5 \mathrm{Fps}$ \\
\hline TETRA 4 slot & $28.8 \mathrm{Kbit} / \mathrm{s}$ & $11.3 \mathrm{Fps}$ & $5.3 \mathrm{Fps}$ & $2.1 \mathrm{Fps}$ \\
\hline WaveLAN & $2 \mathrm{Mbit} / \mathrm{s}$ & $30 \mathrm{Fps}(\mathrm{x} 26)$ & $30 \mathrm{Fps}(\mathrm{x} 12)$ & $30 \mathrm{Fps}(\mathrm{x} 4)$ \\
\hline
\end{tabular}

Table 6: User-selectable profiles for different network types.

The above results allow a set of profiles to be formulated which correspond to different network types. This set of profiles is shown in Table 6 . The table is a relatively simple one that allows frame rate and image size to be traded-off at different network capacities. The frame rates shown are the maximum possible if the network bandwidth is the limiting factor (with a quantization factor of 10). In our current encoding system this is not true and the processing capability of the end-system is the limiting factor (i.e. the encoding rate) In such a case the quantization parameter may be adjusted so that the data stream fills the communication channel, hence producing higher quality images in the stream. From Table 5 it can be seen that reducing the quantization factor may also increase the frame rate. These additional factors may produce a more complex cross-reference of attributes and trade-off options. Error resilience could also add an additional element in a profile table.

In the prototype application described earlier a roaming user may specify a preference for larger image size or faster frame rate depending on the current use of the application. For example, if a security officer were engaged in a WaveLAN supported video call with an image size of QCIF and moved outside the coverage of his local area network then an alternative connection may be established via a slower GSM channel. If the user has specified a preference for a frame rate over image size then the negotiation system would opt to drop the image size to Sub-QCIF and maybe alter the quantization parameters in order to maintain a reasonably similar frame rate.

Table 6 can also be used to estimate the quality a user may expect before establishing a call, and hence chose his medium (or number of channels in TETRA) appropriately.

\section{CONCLUSIONS}

This paper has reported on work carried out to develop prototype mobile multimedia applications to support members of the emergency services. In particular we have described a prototype video surveillance application and its associated infrastructure. These enable groups of collaborating mobile users to access H.263 encoded video streams produced by a number of fixed and mobile cameras. Strategies to deal with video transmission, QoS feedback and late entry are described.

An analysis of our prototype environment has enabled us to produce an experimentally based table of expected video QoS for a range of different networks. This table can be used both to predict the likely QoS the user can expect and to help specify adaptation policies in the face of QoS degradation. A further important result of our work is that we have demonstrated that in the majority of practical cases the quality of H.263 encoded video received by clients is governed by the performance of the end-system performing the compression which in a mobile environment is likely to be of low capability.

Our future work in this area will be to develop a robust version of our application which will be used in a trial with members of the emergency services. This will enable us to obtain subjective feedback on the suitability of very-low-bit-rate encoded video in real application domains.

\section{REFERENCES}

[1] G. Bjontegaard, "Very Low Bitrate Videocoding using H.263 and Foreseen Extensions", Proceedings of European Conference on Multimedia Applications, Services and Techniques (ECMAST '96), Louvain-la-Neuve, Belgium, May 1996, pp 825-838.

[2] D. Clark and D. Tennenhouse, "Architectural Considerations for a New Generation of Protocols", Proceedings of ACM Sigcomm '90, Philadelphia, Pennsylvania, September 1990, pp. 200-208

[3] N. Davies, G. Blair, K. Cheverst and A. Friday, "A Network Emulator to Support the Development of Adaptive Applications", Proceedings of 2nd USENIX symposium on Mobile and Location Independent Computing, Ann Arbur, U.S.A., April 1995.

[4] ETSI Work Programme DE/RES - 0601, Subtechnical Committee (STC) RES 06, "Radio Equipment Systems (RES); Trans-European Trunked Radio (TETRA); Voice plus Data (V+D), Designer's Guide”, May 1995.

[5] Guide context-sensitive mobile multimedia visitor guide, in collaboration with Lancaster Tourist Information, (EPSRC project GL/L05280), further details:

www.lancs.ac.uk/computing/research/mpg/most/guide.html

[6] ISO IEC JTC 1; "Information Technology - Coding of Moving Pictures and Associated Audio for Digital Storage Media up to about $1.5 \mathrm{Mbit} / \mathrm{s}$ ", International Standard (IS) ISO/IEC IS 11172, 1993.

[7] ISO IEC JTC 1; “Information Technology - Coding of audio-visual objets", Working Draft (WD) ISO/IEC 14496-1, 1997.

[8] ITU-T H.261, "Video Codec for Audiovisual Services at px64 kbits", International Telecommunications Union, Telecommunications Standardisation Sector, ITU-T Recommendation H.261, March 1993. 
[9] ITU-T H.263 "Video coding for low bit rate communication" International Telecommunications Union, Telecommunications Standardisation Sector, ITU-T Recommendation H.263 March 1996.

[10] Mobile Multimedia collaborative project with Simoco International Ltd, Barclay Associates Ltd, HW Communications Ltd, (EPSRC LINK PCP project GR/K82024), further details:

www.lancs.ac.uk/computing/research/mpg/most $/ \mathrm{mm} \_$project.html

[11] M. Rahnema, "Overview of the GSM System and Protocol Architecture", IEEE Communication Magazine, Vol. 31, Issue. 4, pp. 92-100, April 1993.

[12] H. Schulzrinne, S. Casner, R. Frederick, V. Jacobson, "RTP: A Transport Protocol for Real-Time Applications", Internet RFC 1889, 01/25/1996.

[13] B. Tuch, "Development of WaveLAN, an ISM band wireless LAN", AT \& T Technical Journal, pp. 27-37, July/August 1993.

[14] N. Yeadon, F. Garcia, D. Hutchison and D. Shepherd, "Filters: QoS Support Mechanisms for Multipeer Communications", IEEE Journal on Selected Areas in Computing (JSAC) special issue on Distributed Multimedia Systems and Technology, Vol. 14, No. 7,. Pp. 1245-1262, September 1996. 